\title{
Condition Monitoring Technologies for Synthetic Fiber Ropes - a Review
}

\author{
Espen Oland ${ }^{1}$, Rune Schlanbusch ${ }^{2}$, and Shaun Falconer ${ }^{2}$ \\ ${ }^{1,2}$ Teknova AS, Kristiansand, Vest-Agder, 4612, Norway \\ espen.oland@uit.no \\ rune.schlanbusch@teknova.no \\ ${ }^{2}$ Department of Engineering and Science, University of Agder, Grimstad, Aust-Agder, 4898, Norway \\ shaun.falconer@uia.no
}

\begin{abstract}
This paper presents a review of different condition monitoring technologies for fiber ropes. Specifically, it presents an overview of the articles and patents on the subject, ranging from the early 70's up until today with the state of the art. Experimental results are also included and discussed in a condition monitoring context, where failure mechanisms and changes in physical parameters give improved insight into the degradation process of fiber ropes. From this review, it is found that automatic width measurement has received surprisingly little attention, and might be a future direction for the development of a continuous condition monitoring system for synthetic fiber ropes.
\end{abstract}

\section{INTRODUCTION}

There is a trend within the offshore market to use fiber ropes instead of steel wire ropes for deep-sea lifting. For steel wire ropes, the weight of the wire itself puts limitations on the deployable depth for the payload, while fiber ropes such as High Modulus Polyethylene (HMPE), which floats in water, allows for the deployment of payloads at deeper depths with smaller cranes. This means that even though fiber ropes are more expensive than steel wires, the cost reductions enter through the reduced size in cranes. Figure 1 shows two recent fiber rope cranes developed by National Oilwell Varco and MacGregor for deep-sea heave-compensated operations. With active heave compensation, the payload will be stationary relative to the seabed, while the rope moves back and forth over multiple sheaves to compensate for the waves, which will cause heat build up in the rope and lead to material deterioration in the fiber rope. The companies are therefore interested in a good condition monitoring technique that can provide the current

Espen Oland et al. This is an open-access article distributed under the terms of the Creative Commons Attribution 3.0 United States License, which permits unrestricted use, distribution, and reproduction in any medium, provided the original author and source are credited.
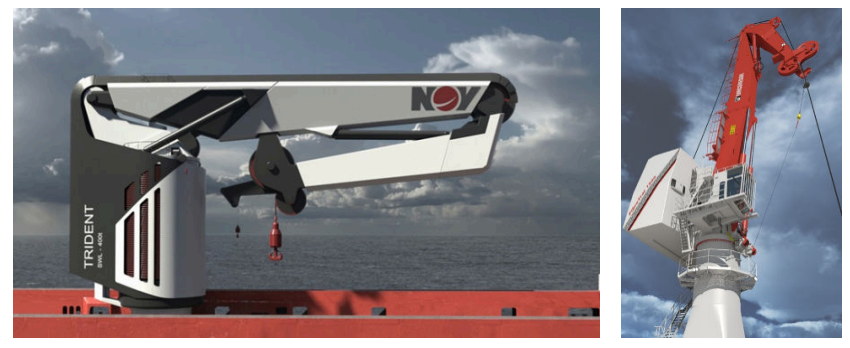

Figure 1. Fiber rope cranes by National Oilwell Varco (left) and MacGregor (right). Reproduced with permissions.

state of the rope, as well as its remaining useful life (RUL).

This paper is an extension of the work on this topic by Oland and Schlanbusch (2016) and presents a review on the available technologies as well as providing recommendations to which technologies to focus on when doing condition monitoring of fiber ropes. Specifically, this paper considers experimental results, new techniques and technologies as well as the different patents on the subject. Previous review papers by Williams and Lee (1982) and Rebel, Chaplin, Groves-Kirkby, and Ridge (2000) have explored condition monitoring techniques of steel wires with the latter containing information about techniques used for fiber ropes. The work by Rebel et al. (2000) has later been expanded by Laura (2003) through a review article on both wire and synthetic ropes with some additional references. Now more than 14 years later, there is a need for a new review on this topic to put the old and new technologies into the same context of condition monitoring of fiber ropes, where also patents are included. Note that there is a more recent review on the topic of fiber ropes by Weller, Johanning, Davies, and Banfield (2015), but their focus is on identification of faults, degradation mechanisms, testing and inspection methods, and not the technologies that can facilitate condition monitoring. 


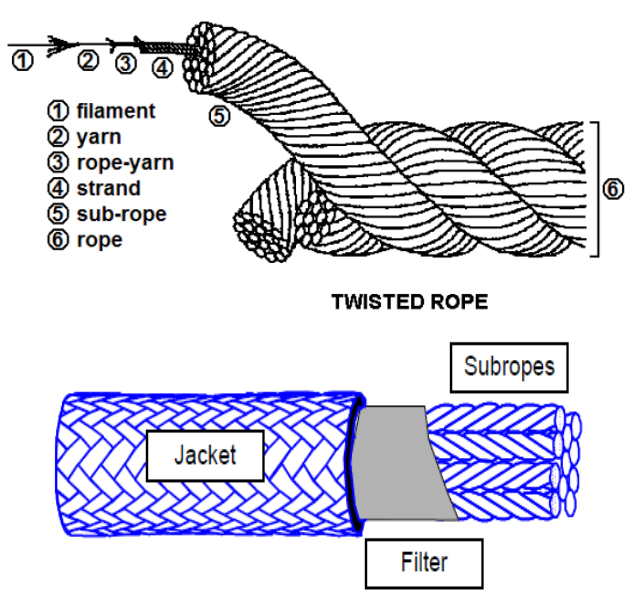

Figure 2. The composite parts of a twisted rope (top), (Lechat et al., 2008) and a parallel sub-rope rope (bottom) (DNV-GL, 2005).

The paper is structured as follows: first the paper presents the construction of a rope and the current practice on when to discard a rope, then it presents the different damage mechanisms common for ropes. The main body of the paper then follows with an overview of the different technologies that facilitates condition monitoring of fiber ropes, before wrapping it up with a discussion on present results and possible future directions.

\section{ROPE PROPERTIES AND CURRENT PRACTICE}

There are many ways of creating a rope, but its basic construction remains the same. Figure 2 shows the construction of a rope, which consists of filaments (continuous fibers), yarns, strands and sub-ropes. Filaments are twisted together to produce yarn, multiple yarns are twisted together to produce a strand, and multiple strands are twisted together to create a sub-rope. Sub-ropes can then be twisted or braided together to form a rope. There are a multitude of different rope designs, ranging from different braiding techniques to parallel sub-rope constructions as well as ropes with or without jackets. Figure 2 (bottom) shows a rope construction of parallel sub-ropes using a jacket. In this case, the jacket is there to keep the load-bearing sub-ropes in place, and does not provide any contribution to the load-bearing capabilities, and the filter is there to protect against ingress of foreign materials. It is important to emphasize that different rope constructions and rope types will naturally require different condition monitoring techniques.

The current practice on when to discard a fiber rope is to do visual inspections and count load cycles. The recommended practice DNV-RP-E304 by DNV-GL describes the process of inspecting a parallel sub-rope rope as in an incremental order: check the jacket, the filter, and then the sub-ropes (DNV-GL,
2005). Minor repairs can be performed on the jacket and filter, but if the sub-ropes are damaged, the number of damaged or severed sub-ropes must be counted and compared to the load-bearing capabilities of the rope. A comparison then dictates whether to discard, repair or continue nominal operation. Another standard DNV-OS-E303, states that any rope that has been subjected to $70 \%$ of its Maximum Break Strength (MBS) should be taken out of service to be re-certified or discarded (DNV-GL, 2013b).

\section{DAMAGE MECHANISMS FOR FIBER ROPES}

The main damage mechanisms for fiber ropes are abrasion, creep, UV radiation, tensile fatigue, heating, compression fatigue, and shock. It is important to consider all of these but it must be noted that the applicability and severity is material dependent. These damage mechanism will be discussed briefly in this section.

Abrasion is defined by Annis (2005) for ASTM as "the wearing away of any part of a material by rubbing against another surface", and there can be both internal and external abrasion. External abrasion can arise due to interactions with e.g. sharp edges, while internal abrasion can arise due to the rubbing between the strands inside the rope. Note that it is the internal abrasion that generates heat in the rope such that the fibers melt together, reducing the strength of the rope. Herduin, Banfield, Weller, Thies, and Johanning (2016) show through experimental tests that there is a linear relationship between the strength loss due to external abrasion and the surface roughness.

Creep is defined by Samson Rope Technologies (2012) as the continued extension of a material when subjected to constant, long-term static loading. It can be reversible or non-reversible depending on the severity of the load history. Figure 3 shows experimental results from 10 load-cycles of a new nylon rope (Weller, Davies, Johanning, \& Banfield, 2013). Due to the load and constructural rearrangement after initial loading, the rope exhibits creep, resulting in a permanent elongation of the rope. Figure 4 shows the three different regimes of an HMPE rope as defined by Vlasblom and Bosman (2006). In Regime I, called "primary creep", the elongation of the rope is reversible such that the rope will return to its original length when the load is removed. In Regime II, called "steady state creep", the molecular chains of the rope start to slide, such that the creep rate increases slightly and the elongation is irreversible. In Regime III, called "tertiary creep", the molecular chains start to break, and there is an increase in local stress that accelerates until breakage. One very important fact regarding creep, is that it is a local phenomenon. This means that the whole rope must be monitored for creep, such that it is not sufficient to monitor the overall elongation of the rope.

Weller et al. (2013) state that UV radiation leads to material degradation of the outer surface of some types of rope, and 


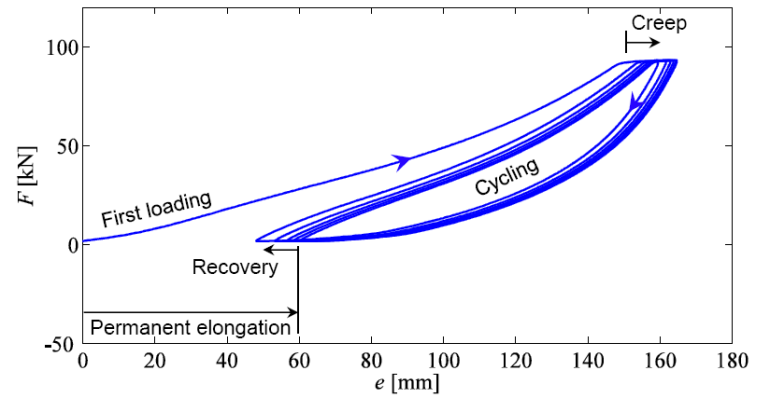

Figure 3. Load extension of a new nylon mooring rope subjected to 10 cycles (Weller et al., 2013).

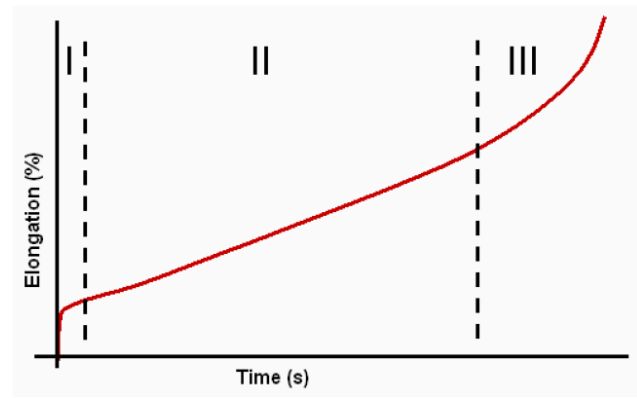

Figure 4. A typical creep curve for HMPE ropes (Vlasblom \& Bosman, 2006).

can be seen as discolored fibers that become weaker than the internal fibers. Flory, McKenna, and Parsey (1982) explain that UV radiation can degrade polypropylene and aramid fibers and to some extent nylon ropes, while polyester and HMPE ropes are unaffected. Furthermore, Flory et al. (1982) wrote that most aramid ropes have a jacket, which protects against UV radiation. The UV radiation also only affects the fibers on the surface on the rope, such that this is mainly a problem for small diameter ropes, and not for large diameter ropes required for deep sea lifting applications.

Tensile fatigue is the result of ropes that have been operated for long periods of time within their operating limits. According to McKenna, Hearle, and O'Hear (2004), the main damage mechanism under this condition is internal fiber-onfiber abrasion, where long-term usage results in a breakdown of yarns. Sea water and salt have an especially accelerating effect on the fatigue of fiber ropes subject to wet/dry cycles due to increased internal abrasion through particle and fiber contact. Kenney, Mandell, and McGarry (1985) state that sea water reduces the strength of nylon ropes by approximately $10 \%$ under most conditions. Salt weakens the nylon structure by disrupting the hydrogen bondings.

Heating is another challenge for fiber ropes, especially with cyclic bend over sheave (CBOS) situations such as active heave compensation, where the rope is moved back and forth over a sheave. This results in a temperature build-up, which will exceed the critical temperature of the rope. For reference, Cortland Company (2016) states that the Cortland Plasma fiber rope brand has a critical temperature of $65^{\circ} \mathrm{C}$. As the temperature increases beyond the critical temperature, the degradation is caused by the temperature alone.

Compression fatigue is defined by McKenna et al. (2004) as when fibers, yarns or strands buckle due to the rope operating at low tension. This leads to a change in the rope such that it exhibits $\mathrm{Z}$ shaped kinks, where the fibers tend to fail at the kink. This is particularly applicable to stiffer materials such as aramid and less-so HMPE.

According to McKenna et al. (2004), shock or overloading a rope above the work load limit can result in a significant loss of strength or durability. Shock can result in melted fibers and repeated shocks is similar to cyclic bending fatigue. The damage might not be possible to detect through inspections, such that McKenna et al. (2004) recommends to use the load history to identify shock damages and to monitor the rope's condition.

It is important to know that each of the different damage mechanisms have an effect on the other damage mechanisms. For CBOS loading in particular, Davies, François, Lacotte, $\mathrm{Vu}$, and Durville (2015) identify four distinct mechanisms through observation of damages:

1. Flattening of rope surface in contact with sheave, producing a shiny continuous surface, which might be due to the local melting of fibers

2. Extrusion of fiber loops, which might be due to the high pressure exerted by the sheave

3. Abrasion within the rope

4. Fiber breakage

Davies et al. (2015) emphasize that these mechanisms are not acting independently, but act together, making failure analysis more complex. This also means that monitoring one parameter alone might not be sufficient to obtain a good condition monitoring system for fiber ropes. Samson Rope Technologies (2014) have documented pictures of many different kinds of rope conditions. Figure 5 shows these conditions, which are: cut strands, compression damage, inconsistent diameter, pulled strands, heavy external abrasion and melted fibers. Note that most of these damages can be monitored through width and surface inspections.

All these damage mechanisms that have been presented here, must be monitored through one or more sensor systems, something that will be discussed in the following section. 

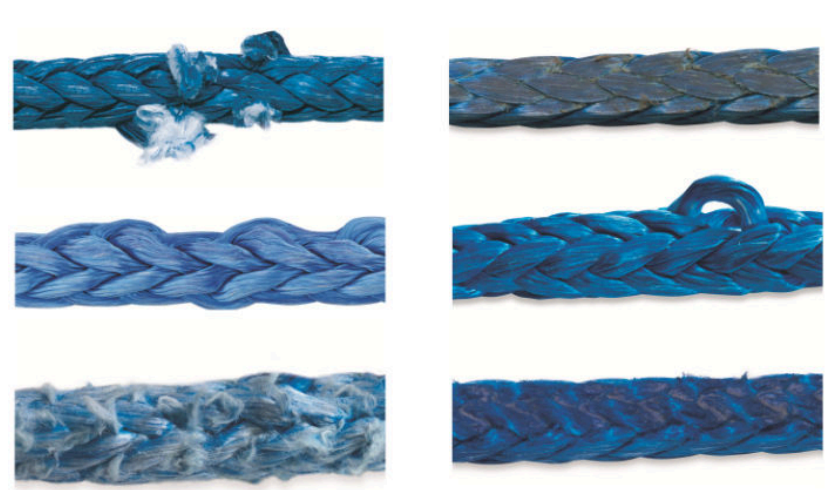

Figure 5. Different kinds of rope damages. Cut strands (top left), compression damage (top right), inconsistent diameter (middle left) and pulled strands (middle right), heavy abrasion (bottom left) and melted fibers (bottom right). Reproduced with permission from Samson Rope Technologies.

\section{TECHNOLOGIES}

In general, there are two main approaches when monitoring the condition of fiber ropes. One is to embed a foreign material or sensor inside the rope, while the other is to measure physical quantities to quantify the condition of the rope. The use of embedded technologies has received much attention, with approaches such as detectable magnetic threads, optical fibers, conductive threads and threads that can be detected using X-ray tomography. These approaches have many advantages, while it requires a specially designed rope to be employed. Also note that it represents a major challenge to implement embedded methods without effecting the rope structure and/or materials. Non-embedded approaches include thermography, computer vision, computed tomography, capacitive detection, acoustic emission and lasers for width measurements. Each of these techniques will be discussed in the following sub-sections.

From an implementation perspective it is important to discuss the applicability of the different sensors for continuous and/or discrete monitoring. Technologies such as conductive threads, optical fibers, thermography, width measurements, capacitive detection, computer vision and acoustic emission can allow continuous monitoring of a rope. Computational expensive sensors such as X-ray tomography and micro-CT tomography will most likely be constrained to measure at discrete intervals (e.g. inspection of the rope on the deck). As such, it is natural to expect that a complete condition monitoring system with prognostic capabilities will comprise a suite of sensors that work together.

\subsection{Magnetic measurements}

While magnetic techniques are common for wire ropes, they are not directly applicable for fiber ropes since they do not consist of ferromagnetic materials. However, there are some

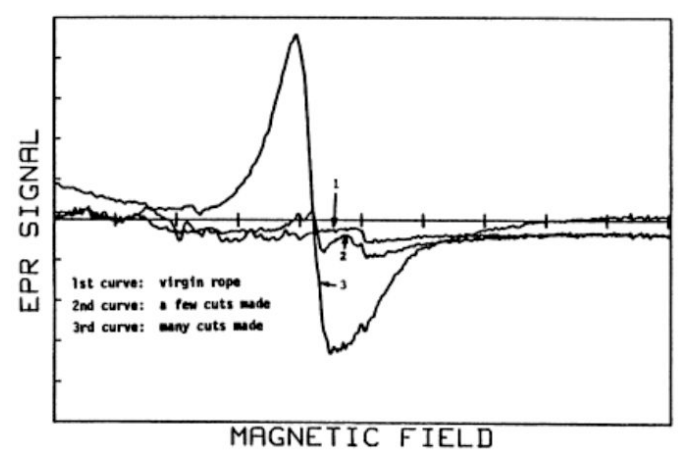

Figure 6. Electron spin resonance traces for virgin and degraded nylon rope (DeNale, 1985). The amplitude increases as a function of cuts.

available methods using magnetic detection techniques that can be applied to fiber ropes.

DeNale (1985) presents an overview of different non-destructive evaluation methods in the U.S. Navy, where the strength of a fiber rope can be determined using electron paramagnetic resonance (EPR) spectroscopy. Through as series of papers (Bryden \& Poehler, 1985), (Bryden \& Poehler, 1986) and (Bryden \& Poehler, 1987) this approach is described in more detail. Irradiation from UV and X-rays as well as mechanical fractures can be used to generate free radicals associated with broken bonds in the polymer chains. These paramagnetic defects can then be detected using EPR spectroscopy by using a broad magnetic field sweep. Figure 6 shows the EPR signal as a function of magnetic flux, where three different cases are considered: a virgin rope, a rope with a few cuts, and a rope with many cuts. The amplitude of the EPR signal increases as a function of cuts, and can therefore serve as a condition indicator for fiber ropes. Note that different ropes will exhibit different behaviors requiring specialized models for condition monitoring.

In a recent patent by Huntley, Huntley, and Whitehill (2015) the inventors propose to make at least one of the strength members detectable by a magnetic sensor. This allows the condition of the rope to be monitored using magnetic flux leakage or eddy currents as the phase and amplitude will change during use. Grabandt, Van Berkel, Oosterhuis, Mathew, and Akker (2015) submitted a similar patent, where a detection thread that is detectable using magnetism, X-ray or terahertz analysis is weaved into the rope. A comparison between the measured pattern and a healthy baseline then enables the determination of the rope's condition.

Huntley, Grabandt, and Graëtan (2015) present an approach called the marked yarn magnetic principle and is similar to the patent by Huntley, Huntley, and Whitehill (2015), where one of the strength members is coated with a marker material that is detectable using magnetism. The received voltage from the 


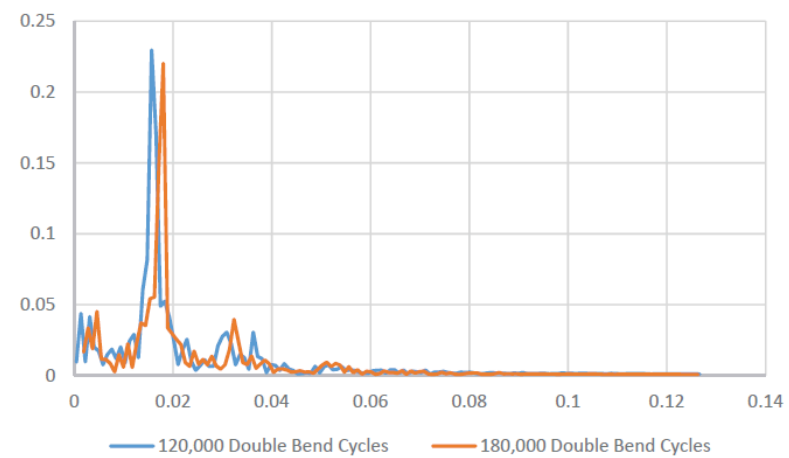

Figure 7. FFT of marked yarn magnetic principle data (Huntley, Grabandt, \& Graëtan, 2015).

sensor can then be related to the position along the rope, facilitating the use of the Fast Fourier Transform (FFT). Figure 7 shows the FFT of the sensor data at 120,000 and 180,000 cycles after a CBOS test. It is evident that the main peak shifts to the right as the number of cycles increases, thereby providing a good condition indicator of damage in the rope.

In their patent, Ilaka and Zerza (2014) specify a detection device for the change in magnetic measurements of an embedded magnetic fiber and compare to user-defined values, which can be used to predict the discard state of specific sections of a rope in conjunction with other parameters.

\subsection{Electrical conductive measurements}

Using embedded conductive wires or threads as part of a composite fiber structure are discussed in several papers (Wang \& Chung, 1997), (Irving \& Thiagarajan, 1998) and (Javidinejad \& Joshi, 1999), which show how to correlate the measured resistance to the fatigue and applied strain respectively. More specifically for ropes, Schmieder, Heinze, and Michael (2015) have developed a rope design that included load-bearing strands with an electrically conductive thread, which allows for condition monitoring. Figure 8 shows the resistance as a function of bending cycles. It is evident that the resistance increases with the number of cycles, something that can serve as a condition indicator for the rope. De Angelis (1998) obtained a patent on using voltage measurements to determine when fiber ropes must be replaced, while Robar, Veronesi, Stucky, and Gieras (2006) have a patent on the use of electrical resistance for detection of rope deterioration.

Since an electric current through a thread induces a magnetic field, the electric thread also allows for using the results on magnetic detection by Huntley, Grabandt, and Graëtan (2015). Also note that the resistance of a wire is a global measurement (between two points), such that it cannot identify the exact location where the rope has been deteriorated, but gives an indication on the overall health of the rope.

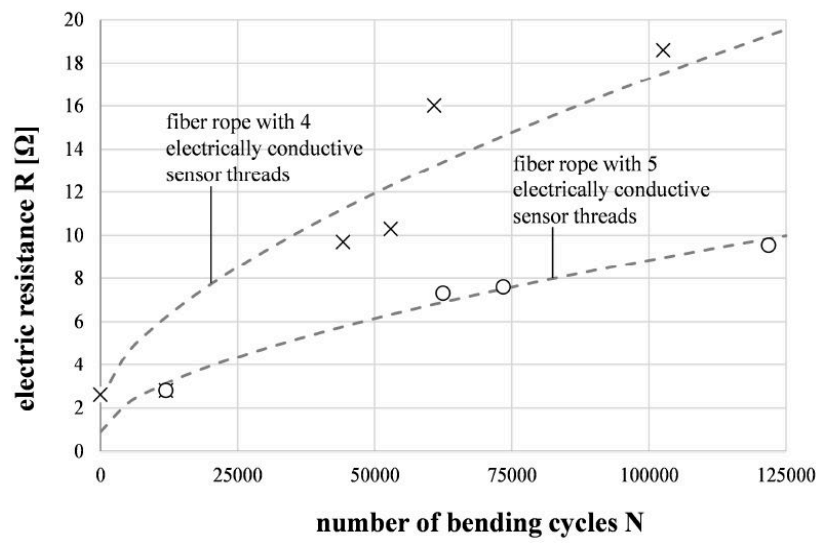

Figure 8. Electrical resistance as a function of bending cycles (Schmieder et al., 2015).

\subsection{Capacitive measurements}

Another method that can be used for condition monitoring of a fiber rope is capacitive measurements. By placing two plates of a parallel capacitor on each side of a rope, the dielectric constant will change as a function of the rope's condition. Grabandt et al. (2015) propose to use this capacitive effect in conjunction with electromagnetic measurements, where the capacitor is one of the frequency determining components, enabling the condition of the rope to be related to the frequency of the received signal. Currently there is little research on this approach beside a few sentences in this patent.

\subsection{Computer vision}

The recommended practice DNV-RP-E304 by DNV-GL recommends visual inspection of ropes for determining their condition (DNV-GL, 2005). A natural extension from manual visual inspection, is the use of computer vision as a means of continuous condition monitoring for fiber ropes.

Törnqvist et al. (2011) performed CBOS testing of a large diameter HMPE fiber rope from Dyneema ${ }^{\circledR}$ with experimental results as shown in Figure 9. During the testing, the authors documented the condition of the fiber rope at $25 \%, 50 \%$, $75 \%$ and $100 \%$ of its lifetime. One of their main observations is that "Generally, at around $50 \%$ of the lifetime, some yarns started to stick out from the rope and about $75 \%$ the strands started to stick out from the rope". This means that computer vision can detect these changes and give indications on the remaining useful life through advanced classification techniques. Even more information about the rope can be obtained by using scanning electron microscopes, which allows detailed study of the abrasion wear and ingress of foreign particles (Weller, Davies, Vickers, \& Johanning, 2015).

Another approach that can work well with computer vision is the use of markings. Schmieder et al. (2015) show that the 

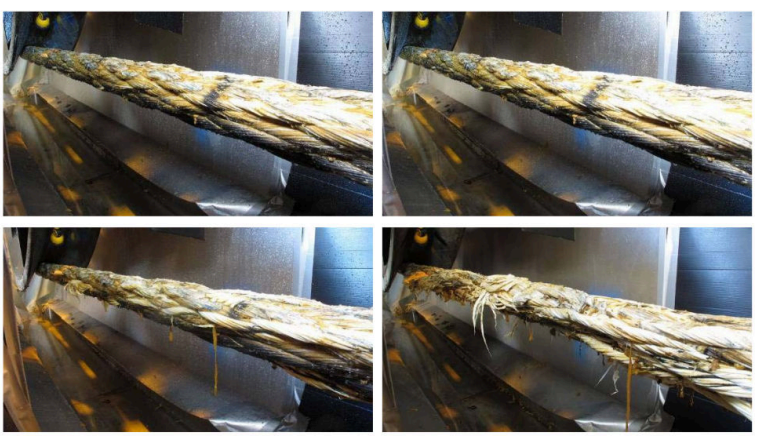

Figure 9. Wear at $25 \%, 50 \%, 75 \%$ and $100 \%$ of the rope lifetime during a CBOS test (Törnqvist et al., 2011).

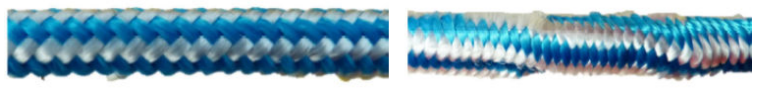

Figure 10. Using longitudinal marks facilitates the detection of damage effects. No damage (left), twist and buckling (right) (Schmieder et al., 2015).

use of markings makes it possible to detect torsion, buckling and relative movement between the core and the jacket. The twist and buckling are mainly exhibited in zones with a low number of CBOS cycles and can be detected using computer vision. Figure 10 shows how to identify the twist and buckling. Markings can also be used to detect changes in length. In addition to this, Ilaka and Zerza (2014) make reference to the use of markers to separate rope sections in their device for gauging the discard state of fiber ropes.

The use of markings on ropes is not new, and has previously been patented, especially for use in elevators. Logan, Favrow, Haas, Stucky, and Baldwin (2006) have a patent where the inventors propose to use markings on a belt, and use changes in the markings as condition indicators for the load bearing members. De Angelis (1999) is another inventor who proposes to use a torsional neutral rope construction. If the rope is weakened by unequal wear and external influences, the rope will begin to twist, which will be detectable by studying the changes in colored markings. These represent good condition indicators that can serve in conjunction with other sensors.

\subsection{Thermography}

The temperature is a measurement that provides vital information of the rope's condition. Davies et al. (2013) state in a recent paper: "...it is the internal abrasion rather than the external pulley/sheave interactions which will govern the rope lifetime". This means that it is of critical importance to monitor the internal temperature inside the rope, as internal abrasion leads to an increase in temperature. Figure 11 shows a comparison between the energy dissipated due to internal abrasion and the energy dissipated from interactions with the sheave. It is evident that the internal abrasion is the main contributor to the increase in temperature. Törnqvist et al. (2011) support this statement, as they observed that the higher temperatures were between the strands, mainly due to friction from the internal movement.

In DNV-RP-E305, a recommended practice for design, testing and analysis of offshore fiber ropes, the authors present a parameter they call the 3-T (DNV-GL, 2015). It is a composite parameter comprising the critical parameters tension, temperature and time. Each parameter depends on the other two and can be treated as a three-dimensional critical parameter called 3-T, which is also described in DNV-GL (2013a). By evaluating the logarithmic time-to-rupture as a function of tension at a given temperature, the authors are able to obtain a linear relationship between tension and time-to-rupture. This can naturally be extended to surfaces that can describe the condition of the rope, and highlights the importance of temperature measurements. A good starting point for doing the required thermal modeling can be found through the works (Bosman, 1996) and (Overington \& Leech, 1997).

De Angelis (2002) obtained a patent on thermal monitoring of fiber ropes where the inventor proposes to embed a conductive temperature element, which facilitates condition monitoring of the rope. If the rope exceeds a critical temperature, a checking circuit can provide a warning signal to the operator.

\subsection{Computed tomography}

$\mathrm{X}$-ray computed tomography provides increased insight into what happens with a rope during use. Davies et al. (2013) performed a CBOS test with a fiber rope where they performed $\mathrm{X}$-ray imaging before and after testing. Figure 12 shows the $\mathrm{X}$-ray images, where it can be observed that the fibers melt together during use, creating a denser rope. This can be detectable using X-ray computed tomography together with advanced classification techniques. The rope will also exhibit changes in how the temperature propagates, something that can be detected using thermography. In their work Schmieder et al. (2015) and Schmieder, Heinze, and Michael (2016), the authors also produced X-ray images during CBOS testing. Figure 12 (right subfigure) shows the longitudinal direction of this rope, where it can be observed that internal abrasion makes the rope thinner.

Huntley, Grabandt, and Graëtan (2015) recently presented results on X-ray imaging. Figure 13 shows a fiber rope where a strength member that is detectable by X-ray is included. The left subfigure shows a part of the rope that is not in the bend zone, where the detectable strength member remains continuous throughout the section. The right subfigure shows a part of the rope that has been exposed to 46,000 bend cycles, where the strength member becomes discontinuous and deteriorated. This means that the X-rays can provide very good 

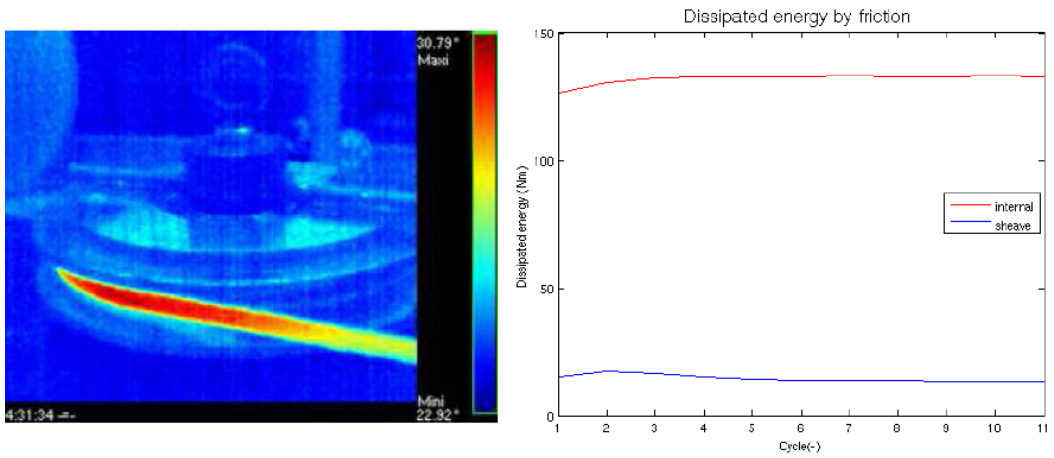

Figure 11. Infrared (IR) image during CBOS testing (left) and dissipated energy as a function of cycles (right) (Davies et al., 2013). The red line is the dissipated energy through internal friction, while the blue line is the energy dissipated through external friction.
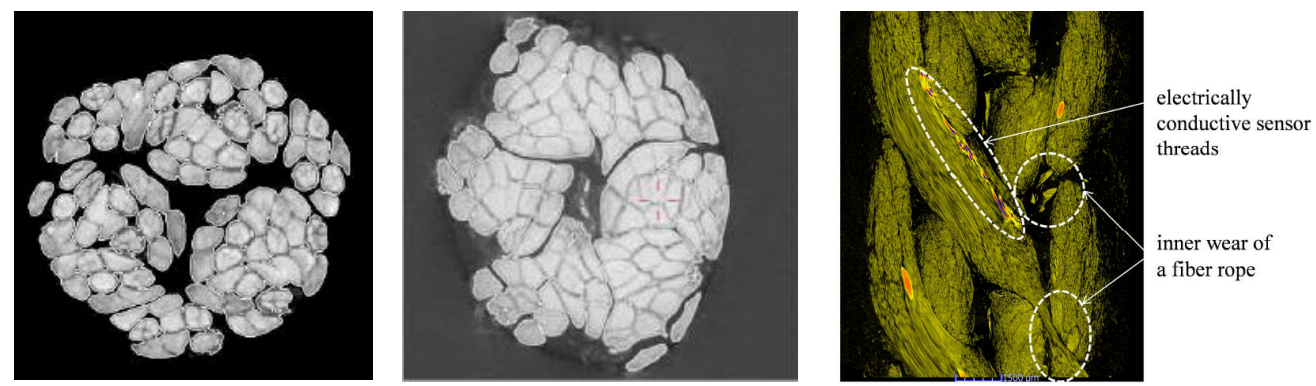

Figure 12. X-ray tomography of a braided fiber rope before (left) and after (middle) testing (Davies et al., 2013). The subfigures are not to scale. The right subfigure shows the longitudinal direction of a worn rope (Schmieder et al., 2015).
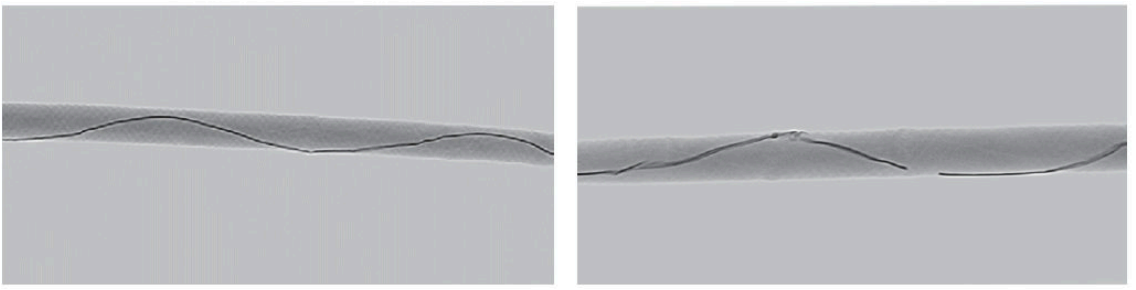

Figure 13. X-ray image of a fiber rope with a detectable strength member. The left picture shows an undamaged section, while the right picture shows a section that has been exposed to cyclic bending (Huntley, Grabandt, \& Graëtan, 2015). 
information on the overall health of the rope in question. Also note that the authors were able to provide an excellent correlation between the X-ray results and the marked yarn magnetic detection principle.

Toda, Grabowska, and Ciesielska-Wrobel (2016) present another application of X-ray tomography applied to fiber ropes. The authors use micro-tomography to measure the parameters of the internal structure of the rope and were able to find the tension generated in the yarns as well as the unevenness of the twist. Additionally, they obtained the distributions of fibers in 3D, facilitating classification of number of fibers as a function of distance from the center of the rope. Figure 14 shows the cross section of the rope as well as the quantity of fibers as a function of distance. This gives excellent information that can be used for condition monitoring, and allows for the monitoring of the internal abrasion, which is known to govern the lifetime of the rope. Additionally, the thermal properties of the rope can be calculated through the knowledge of the fibers and their friction coefficients.

Sand and salt inside the rope result in increased internal abrasion and must be accounted for when doing offshore operations. Schecklman, Kniffin, and Zurk (2014) presented recently a paper on using Terahertz non-destructive evaluation of nylon ropes, where the authors were able to map the $3 \mathrm{D}$ distribution of sand and salt contaminants within the rope, thereby providing a good condition indicator for internal abrasion due to these contaminants.

\subsection{Optical fibers}

Optical fibers are able to measure parameters such as strain, temperature, acceleration and acoustic emissions, and therefore have a wide range of applications and are very popular for smart structures.

Ractliffe (1982) patented one of the first designs using optical fibers for condition monitoring of fiber ropes. The inventor proposes to embed optical fibers into the strands of a fiber rope to provide continuous monitoring, and to identify high local strains. D'Agostino, Barrick, and Williams (1993) propose another design through a patent where the strains and stresses are determined by monitoring the fiber light transmissive and reflective properties. The inventors propose to use a reference pattern measured when the rope is new, and compare it with patterns measured during use. The changes in patterns over time then serves as condition indicators.

In two separate papers, (Cortázar, Tomasel, \& Laura, 1998) and (Cortázar, Lorrondo, Laura, \& Avalos, 1996), the authors present experimental results on using optical fibers for condition monitoring of Kevlar rope and on steel wire ropes. Figure 15 shows the rupture signals for both synthetic and steel wire rope, where it is evident that the shape of the received signals using optical fibers are very similar for both kinds of rope. This highlights the advantages of using optical fibers, as they can be applied to both steel wire and synthetic rope.

Ludden, Carroll, and Burgoyne (1995) present another approach of using optical fibers for condition monitoring of fiber ropes. The authors show that by using optical time domain reflectometry (OTDR), they are able to detect the acoustic emissions caused by yarn snapping. By counting the number of yarn snappings, it allows for the quantification of the number of broken yarns as a function of distance to determine where the snapping has taken place, thereby providing critical information on the condition of the fiber rope. Robertson and Ludden (1997) augmented this result by including strain and temperature measurements using fiber Bragg grating pairs spaced along the fiber with localization using wavelength tunable OTDR. Fiber Bragg gratings work by embedding several gratings into the structure of the optical fiber. Each grating will reflect light on a specific wavelength and the reflected wavelengths will change depending on the strain experienced by each grating, thereby facilitating strain measurements. Note that with a limited spectrum, there is a maximum number of gratings for each optical fiber, such that multiple optical fibers are required to obtain sufficient resolution (especially important for deep sea operations with rope lengths of several kilometers).

Rebel et al. (2000) performed a review on different condition monitoring techniques for fiber ropes, where they deemed the use of fiber optics using Bragg gratings as the most promising approach for condition monitoring of mooring ropes. The authors propose to embed multiple optical fibers in a helix trajectory in the fiber rope to provide sufficient resolution of both strain and temperature measurements, while avoiding the breakage of the optical fibers due to the strain on the rope itself.

Barton Smith and Williams (2002) measured the strain in mooring ropes using optical fibers. The authors propose to embed an optical fiber into each of the seven subropes and use OTDR to measure the strain. Through experiments the authors obtained a linear relationship between the applied and measured strain.

Another approach for fiber optics is by using Brillouin scattering. Brillouin scattered light occurs due to an interaction with acoustic waves and results in a frequency shift in the scattered light. The Brillouin frequency shift is the difference between the incident and scattered light frequency, is proportional to the temperature, and can be used for strain measurements.

Thévanaz, Niklès, Fellay, Facchini, and Robert (1998) and Uttamchandani et al. (1999) respectively, presented papers on using the Brillouin scattering for strain measurements in synthetic ropes. In the latter paper it is highlighted that more research was required on this topic for it to be a viable solu- 

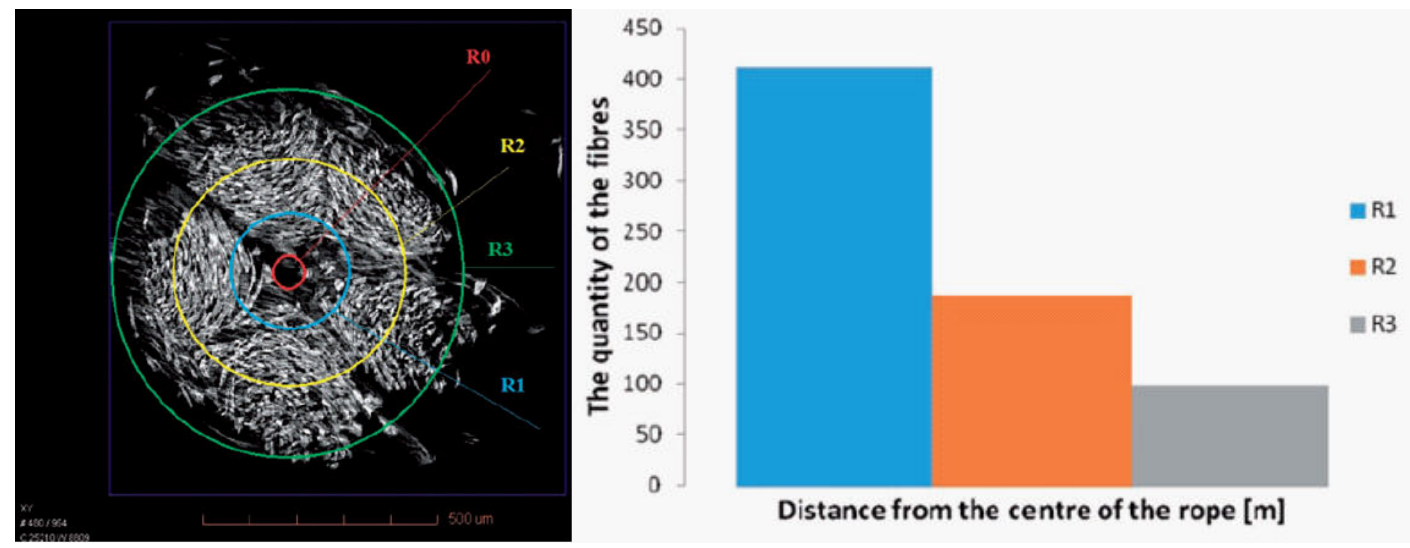

Figure 14. Cross section of the rope (left) and distribution of fibers as a function of distance (right) (Toda et al., 2016).
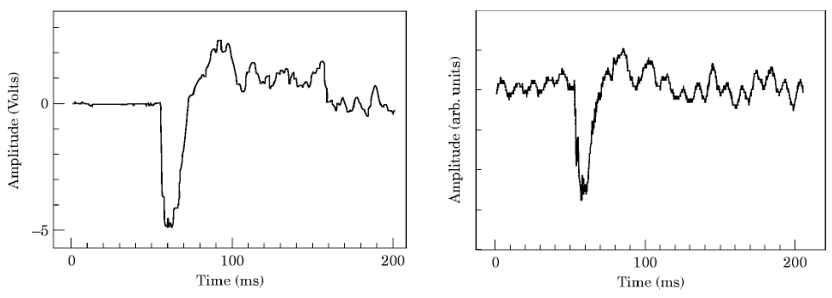

Figure 15. Typical rupture signals for synthetic rope (left) and steel wire rope (right) (Cortázar et al., 1998). Notice the similarity of the two curves.
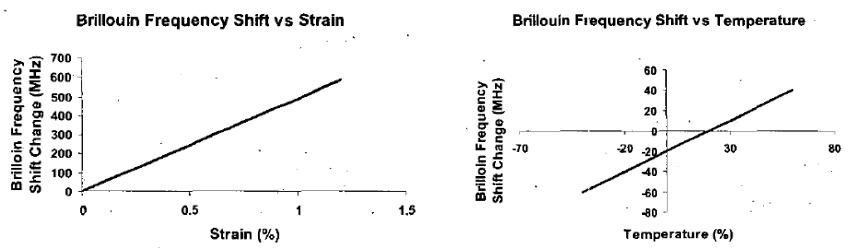

Figure 16. Relationship between the Brillouin frequency shift, strain and temperature (O'Hear, 2003).

tion. O'Hear (2003) presented results on the same technique, where the author was able to obtain a linear relationship between the strain and the Brillouin frequency shift. Figure 16 shows this relationship. Even though it is often argued that it is challenging to discriminate between the temperature and strain effect using Brillouin shift measurements, Thévanaz et al. (1998) emphasizes that this can easily be worked out, however bending and torsion will also need to be accounted for as they will affect the transmissivity of the optical fibers.

\subsection{Vibration and acoustic emission}

The use of ultrasonic and acoustic emission (AE) for condition monitoring of fiber ropes date back to the early 70's. Williams and Lee (1982) did a short review in 1982 on non- destructive testing (NDT) of ropes (both fiber and steel wire), where they focused on acoustic emission techniques. Beside the references on wire ropes, only three papers on fiber ropes were cited. Vanderveldt and Tran (1971) apply the stress wave emission monitoring technique to synthetic ropes and show that there is a significant increase in the acoustic emission rate at about $85 \%$ of the failure load. Egan (1972) showed that by using multiple accelerometers mounted onto the rope, the AE signal behaved randomly until about 60$70 \%$ of the rope breaking strength. Above this level, the author was able to pinpoint the region of rupture from the AE signal. Fresch, Yeager, and Thiruvengadam (1979) studied the internal friction damping of virgin ropes and overstressed ropes. The authors were able to show that the damping of virgin ropes were higher than that of the overstressed ropes, and showed a correlation between the damping and the rope breaking strength. Yeager, Hochrein, and Sherrard (1982) evaluated the internal friction damping technique for marine application in 1982 .

Williams, Hainsworth, and Lee (1984) also published a paper on using acoustic ultrasonic NDT to evaluate the condition of fiber ropes. The authors fixed a transmitter and a receiver onto a rope in tension and measured the Stress Wave Factor (SWF). The authors were able to correlate the received signal to the load, but also emphasized that good SWF modeling is required for the technique to be applicable for NDT.

Winter and Green (1985) propose to use mechanical spectroscopy for condition monitoring of fiber ropes, where the authors studied the wave propagation characteristics of a rope under tension. The authors were able to identify differences between new and used ropes, but the frequency response curves were found to change with time under constant load.

Kwun and Burkhardt (1989) continued with this work through their paper where they used the transverse impulse vibration method to study the condition of the rope. The method is able 


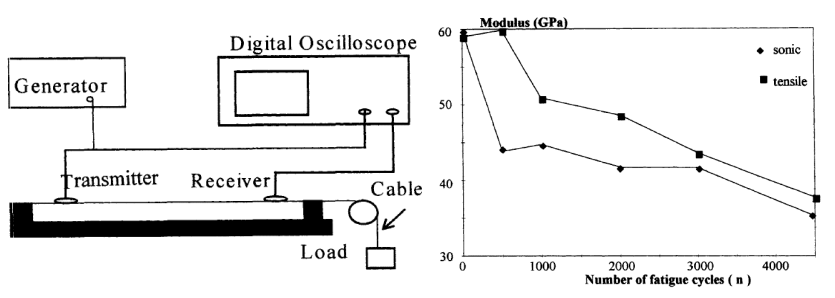

Figure 17. Experimental setup (left) and the dynamic modulus as a function of cycles (right) (Ferreira et al., 2000).

to detect localized flaws in the rope, and uses a similar setup as (Williams et al., 1984) with a transmitter and a receiver on a rope in tension. Their work later resulted in a patent and an article produced by Kwun and Burkhardt (1990) and Kwun and Burkhardt (1991) respectively.

Ferreira, Lam, Koncar, and Delvael (2000) studied the dynamic modulus of a fiber rope by using longitudinal wave propagation. The modulus describes the elastic properties of the rope and has the same behavior as the fatigue of the rope. By measuring the propagation speed along the rope and knowing the density of the rope, the modulus can be determined and serve as a condition indicator. Figure 17 shows the setup as well as their test results. It is evident that there is a correlation between the modulus and the deterioration of the rope. Smith, Nickerson, Palazzola, and Pawrrish (2005) obtained a patent on this approach for evaluating the condition of aramid fiber ropes used in elevators.

The results by Ferreira et al. (2000) and Kwun and Burkhardt (1991) can be extended to become non-contact by using laser scanning vibrometry. Laura, Ercoli, and Malfa (1995) presented a setup in a paper where the authors used an infrared emitter diode and a receiver diode to monitor the vibration of a mechanical cable (applicable to both steel wire and synthetic rope). The basic principle is that the infrared incident ray is reflected by the cable where the vibration modifies the reflected ray. This allows for the determination of the frequency of the cable, and facilitates contact-less vibration measurements. Figure 18 shows the experimental set-up by Laura et al. (1995). In addition to using the infrared measurement technique, the authors mounted accelerometers onto the cable, and were able to obtain a good correlation between the infrared technique and accelerometer-based measurements.

In a recent patent by Padilla, Bull, Royer, and Owens (2015), the inventors propose a contact-less solution where a transmitter and a receiver are mounted on two sides of a fiber rope, and then monitor the received amplitude and propagation time. During their experimental testing, the synthetic rope broke after 3800 cycles. Figure 19 shows their main results where it is evident that these trends can serve as condition indicators for determining the remaining useful life of the fiber rope. The recent work by Bashir et al. (2017) support

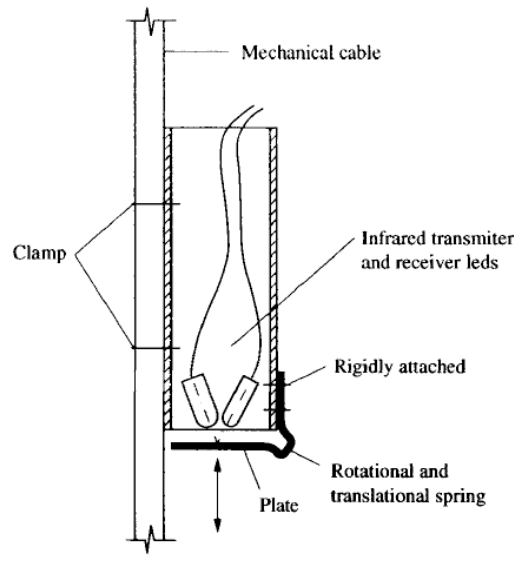

Figure 18. Infrared transducer set-up (Laura et al., 1995).
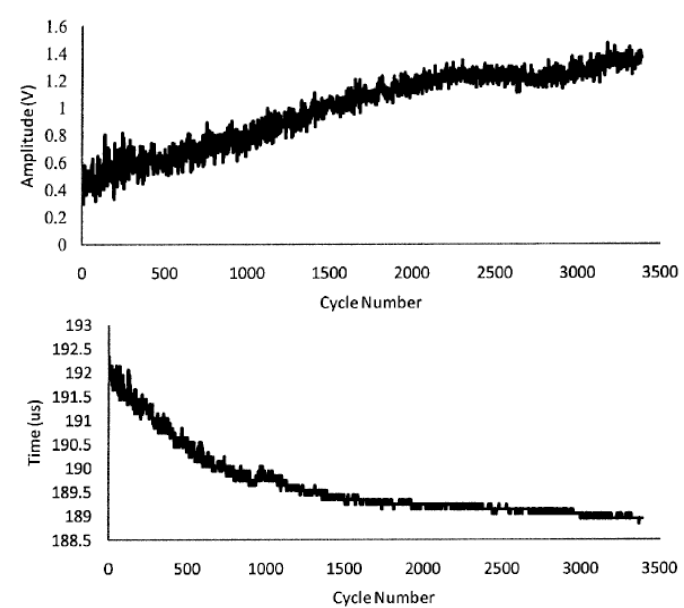

Figure 19. Experimental results using acoustics. Received signal amplitude (top) and propagation time (bottom) as functions of cycles (Padilla et al., 2015).

this, as the authors are able to obtain consistent experimental results using acoustic emission to monitor the condition of mooring ropes.

\subsection{Diameter measurements}

Width measurements using lasers, optical cameras, or other technologies, can provide vital inputs to the condition of the rope. Especially when considering ropes that exhibit creep behavior, it follows that as the rope becomes longer its width is decreased due to a positive Poisson's ratio. Hence, by monitoring the width alone will give very important information on the rope.

Samson Rope Technologies (2014) state that a rope can have cut strands, compression damage, inconsistent diameter, pulled strands, heavy abrasion or melted fibers as shown in Figure 5. Each of these damages (except melted fibers) are de- 
tectable by monitoring the diameter of the rope, emphasizing the importance of diameter measurements. Through a personal discussion with Nick O'Hear (May 12th, 2016), he recommended to focus on width measurements when doing condition monitoring of fiber ropes. Currently there is little open research on using diameter measurements for condition monitoring of fiber ropes. However, in a recent patent application by Van Der Woude and Zijlmans (2015), the inventors propose to correlate physical measurements (diameter of the rope, shape of the rope, elongation, creep, bending, tension and temperature) to the position along the rope. As an example, the inventors show how the diameter of a steel wire rope can be related to the position along the rope, providing vital information on the condition of the rope.

The patent by Ilaka and Zerza (2014) makes use of a clamping roller set up attached to springs. The lower set of these sprung rollers has a rotary axle and lever where the measured diameter is transferred to a position sensor, allowing cross sectional changes to be detected. In this system the rope is split into sections, allowing the changes to be monitored and compared to a previously user-defined discard criteria which will send a warning to the operator indicating the rope section must be replaced. Position sensors are also used in this system to detect changes in length of the rope in conjunction with the decrease in diameter.

\section{Conclusion AND Discussion}

This paper has presented an overview of the different technologies facilitating condition monitoring of fiber ropes. From the recent trends, the work by Huntley, Grabandt, and Graëtan (2015) and Huntley, Huntley, and Whitehill (2015) show a promising method of embedding a strength member detectable using either magnetism or X-ray imaging. This shows that it is possible to obtain high quality information through embedding foreign bodies into the rope.

Thermography is another interesting direction. The work by De Angelis (2002) can be expanded upon or used together with IR imaging for determining the rope's condition. One of the main questions that can serve as a good condition indicator is: "What is the change in temperature as a point on the rope moves away from the sheave?" Figure 12 shows that the structural properties of the rope is changed during use, something that will affect the thermal conductivity of the rope. This might be a good direction for future research.

The patent by Padilla et al. (2015) using acoustic emission shows a clear correlation between the received amplitude and propagation time as functions of cycles. The received amplitude increases about $0.5 \mathrm{~V}$ from 0 to 3500 cycles. In their experimental results, the inventors considered an HMPE rope exposed to 6 cycles per minute. With the knowledge that creep is one of the dominant failure mechanisms for HMPE ropes, it follows that the HMPE rope becomes longer, and hence its width is reduced. This means that the results by Padilla et al. (2015) might be just another way of measuring the width of the rope. As stated before, there are not many results on automatic width measurements in the open literature, such that this represents perhaps one of the most interesting future direction within condition monitoring of fiber ropes.

For subsea ropes, biofouling can make width measurements challenging. This becomes especially critical for mooring ropes, and a method for removing or accounting for this major noise distortion must be considered before applying width measurements as a condition indicator. Other technologies such as thermography (using an IR camera), wave propagation, capacitive measurements will also face challenges subsea due to damping in water and biofouling. For lifting application, it is recommended to place the sensors on the crane. This can provide continuous measurements for parts of the rope, and discrete measurements for the whole rope when the rope is reeled in.

While there are several different sensors that give indications on the condition of the rope, no single measurement will be sufficient to cover all the different failure modes. Inputs to prognostic algorithms can for example be a combination of width, length, temperature, load history and optical images that together might provide accurate estimates on the rope's condition and remaining useful life.

\section{ACKNOWLEDGMENT}

This project is funded through the Norwegian Research Council, SFI Offshore Mechatronics, project number 237896. The authors would like to thank the anonymous reviewers for the excellent feedbacks that helped improve this paper.

\section{REFERENCES}

Annis, P. A. (2005). Surface wear analysis of fabrics. ASTM Standardization News.

Barton Smith, D., \& Williams, J. (2002). Direct measurement of large strains in synthetic fiber mooring ropes using polymeric optical fibers. In Proceedings of the offshore technology conference. Houston, Texas, USA.

Bashir, I., Walsh, J., Thies, P. R., Weller, S. D., Blondel, P., \& Johanning, L. (2017). Underwater acoustic emission monitoring - experimental investigation and acoustic signature recognition of synthetic mooring ropes. Applied Acoustics, 121, 95-103.

Bosman, R. L. M. (1996). On the origin of heat build-up in polyester ropes. In Proceedings of OCEANS. FL, USA.

Bryden, W. A., \& Poehler, T. O. (1985). Non-destructive testing of nylon ropes using magnetic resonance techniques. In Proceedings of the 11th world conference on non-destructive testing. Columbus, Ohio, USA. 
Bryden, W. A., \& Poehler, T. O. (1986). NDT of nylon ropes using magnetic resonance techniques. Review of Progress in Quantitative Non-Destructive Evaluation, 5A, 1393-1396.

Bryden, W. A., \& Poehler, T. O. (1987). EPR imaging investigation of damage in polymeric solids. Review of Progress in Quantitative Nondestructive Evaluation, 7A, 295-298.

Cortázar, O. D., Lorrondo, H. A., Laura, P. A. A., \& Avalos, D. R. (1996). A low-cost fiber optic system for monitoring the state of structural health of a mechanical cable. Ocean Engineering, 23(2), 193-199.

Cortázar, O. D., Tomasel, F. G., \& Laura, P. A. A. (1998). Monitoring the structural health of kevlar cables by means of fiber-optic technology. Journal of Sound and Vibration, 214(3), 576-579.

Cortland Company. (2016). Plasma 12 strand tech sheet. http://www.cortlandcompany.com/plasma (accessed 10th of August 2016).

D’Agostino, W. L., Barrick, M. D., \& Williams, G. R. (1993). Device, system and process for detecting tensile loads on a rope having an optical fiber incorporated therein. Patent US 5182779 A, USA.

Davies, P., François, M., Lacotte, N., Vu, T. D., \& Durville, D. (2015). An empirical model to predict the lifetime of braided HMPE handling ropes under cyclic bend over sheave (CBOS) loading. Ocean Engineering, 97, 74-81.

Davies, P., Lacotte, N., Kibsgaard, G., Craig, R., Cannell, D., Francois, S., ... Sherman, D. (2013). Bend over sheave durability of fibre ropes for deep sea handling operations. In Proceedings of the ASME 2013 32nd international conference on ocean, offshore and arctic engineering. Nantes, France.

De Angelis, C. (1998). Equipment for determining when synthetic fiber cables are ready to be replaced. Patent US 5834942 A, USA.

De Angelis, C. (1999). Apparatus for identification of need to replace synthetic ropes. Patent US 6247359 B1, USA.

De Angelis, C. (2002). Synthetic fiber cable with temperature sensor. Patent US 6392551 B2, USA.

DeNale, R. (1985). Solid mechanics research for quantitative non-destructive evaluation. In Proceedings of the ONR symposium on solid mechanics research for $Q N D E$. Northwestern University, Evanston, IL, USA.

DNV-GL. (2005). Damage assessment of fibre ropes for offshore mooring. DNV-RP-E304.

DNV-GL. (2013a). Manufacturers of offshore fibre yarns. Approval of Manufacturers No. 322.

DNV-GL. (2013b). Offshore fibre ropes. DNV-OS-E303.

DNV-GL. (2015). Design, testing and analysis of offshore fibre ropes. DNVGL-RP-E305.

Egan, D. M. (1972). An acoustic emission study of the fracture mechanism of synthetic rope - an adventure in rhe- ology. In Academy scholars report. U. S. Coast Guard Academy, New London, Connecticut, USA.

Ferreira, M., Lam, T. M., Koncar, V., \& Delvael, Y. (2000). Nondestructive testing of polyaramide cables by longitudinal wave propagation: study of the dynamic modulus. Polymer Engineering and Science, 40(7), 16281634.

Flory, J. F., McKenna, H. A., \& Parsey, M. R. (1982). Fiber ropes for ocean engineering in the 21 st century. In Proceedings of the civil engineering in the oceans. American Society of Civil Engineers, San Diego, CA, USA.

Fresch, D. C., Yeager, L. L., \& Thiruvengadam, A. P. (1979). Engineering feasibility of internal friction damping as a nondestructive evaluation technique for synthetic ropes. Report CG-D-51-79, Springlake Research Center.

Grabandt, O., Van Berkel, B., Oosterhuis, F., Mathew, T., \& Akker, P. G. (2015). Method for non-destructive testing of synthetic ropes and rope suitable for use therein. Patent US 20150225894 A1, USA.

Herduin, M., Banfield, S., Weller, S. D., Thies, P. R., \& Johanning, L. (2016). Abrasion process between a fibre mooring line and a corroded steel element during the transit and commissioning of a marine renewable energy device. Engineering Failure Analysis, 60, 137154.

Huntley, E. W., Grabandt, O., \& Graëtan, R. (2015). Nondestructive test methods for high-performance synthetic rope. In Proceedings of the 5th international OIPEEC conference. Stuttgart, Germany.

Huntley, E. W., Huntley, M. B., \& Whitehill, A. S. (2015). Synthetic rope, fiber optic cable and method for nondestructive testing thereof. Patent US 9075022 B2, USA.

Ilaka, M., \& Zerza, H. (2014). Apparatus for recognizing the discard state of a high-strength fiber rope in use in lifting gear. Patent US 2014/0027401 A1, USA.

Irving, P. E., \& Thiagarajan, C. (1998). Fatigue damage characterization in carbon fibre composite materials using an electrical potential technique. Smart Materials and Structures, 7(4), 456-466.

Javidinejad, A., \& Joshi, S. (1999). Design and structural testing of smart composite structures with embedded conductive thermoplastic film. Smart Materials and Structures, 8, 585-590.

Kenney, M., Mandell, J., \& McGarry, F. (1985). The effects of sea water and concentrated salt solutions on the fatigue of nylon 6,6 fibres. Journal of Materials Science, 20(6), 2060-2070.

Kwun, H., \& Burkhardt, G. L. (1989). Nondestructive testing of ropes using the transverse impulse vibration method. Review of Progress in Quantitative Nondestructive Evaluation, 8, 1053-1060.

Kwun, H., \& Burkhardt, G. L. (1990). Non-destructive eval- 
uation of ropes by using transverse impulse vibrational wave method. Patent US 4979125, USA.

Kwun, H., \& Burkhardt, G. L. (1991). Relationship between reflected signal amplitude and defect size in rope inspection using a transverse-impulse vibrational wave. NDT \& E International, 24(6), 317-319.

Laura, P. A. A. (2003). Monitoring the structural condition of cables: experimental methods. Mechanics, 32(1-2), 10-20.

Laura, P. A. A., Ercoli, L., \& Malfa, S. L. (1995). Feasibility of detection of cable failure using infrared technology. Ocean Engineering, 22(6), 545-549.

Lechat, C., Bunsell, A. R., Davies, P., \& Burgoyne, C. J. (2008). Characterisation of long term behaviour of polyester fibres and fibre assemblies for offshore mooring lines. In Proceedings of oilfield engineering with polymers. Cavendish Conference Centre London, UK.

Logan, D. E., Favrow, L. H., Haas, R. J., Stucky, P. A., \& Baldwin, N. R. (2006). Load bearing member for use in an elevator system having external markings for indicating a condition of the assembly. Patent US $7117981 \mathrm{~B} 2$, USA.

Ludden, B. P., Carroll, J. E., \& Burgoyne, C. J. (1995). A distributed optical fibre sensor for offshore applications. In Proceedings of the IEE electronics division colloquium on opticaltechniques for structural monitoring. London,UK.

McKenna, H. A., Hearle, J. W. S., \& O’Hear, N. (2004). Handbook of fibre rope technology. Woodhead publishing, ISBN: 978-1-85573-606-1.

O'Hear, N. (2003). Optical scanning apparatus for ropes nondestructive test monitoring system. In Proceedings of OCEANS. San Diego, CA, USA.

Oland, E., \& Schlanbusch, R. (2016). A review of condition monitoring techniques for fiber ropes. In Proceedings of the joint conference: Machinery failure prevention technology (MFPT) and ISAs 62nd international instrumentation symposium. Dayton, USA.

Overington, M. S., \& Leech, C. M. (1997). Modelling heat buildup in large polyester ropes. International Journal of Offshore and Polar Engineering, 7(1).

Padilla, L. S., Bull, P., Royer, R. L., \& Owens, S. E. (2015). Non-contact acoustic signal propagation property evaluation of synthetic fiber rope. Patent US $8,958,994$ B2.

Ractliffe, A. T. (1982). Synthetic fibre rope: monitoring strain using optical fibre. Patent GB 2130611 A, UK.

Rebel, G., Chaplin, C. R., Groves-Kirkby, C., \& Ridge, I. M. L. (2000). Condition monitoring techniques for fibre mooring ropes. Insight: Non-Destructive Testing and Condition Monitoring, 42(6), 384-390.

Robar, T. M., Veronesi, W. A., Stucky, P. A., \& Gieras, J. F. (2006). Method and apparatus for detecting elevator rope degradation using electrical resistance. Patent US
7123030 B2, USA.

Robertson, P. A., \& Ludden, B. P. (1997). A fibre optic distributed sensor system for condition monitoring of synthetic ropes. In Proceedings of the IEE colloquium on optical techniques for smart structures and structural monitoring. London, UK.

Samson Rope Technologies. (2012). Understanding creep. Samson Technical Bulletin.

Samson Rope Technologies. (2014). Rope user's manual guide to rope selection, handling, inspection and retirement. Presentation.

Schecklman, S., Kniffin, G. P., \& Zurk, L. M. (2014). Terahertz non-destructive evaluation of textile ropes and slings. In Proceedings of the 2014 international symposium on optomechatronic technologies. Washington DC, USA.

Schmieder, A., Heinze, T., \& Michael, M. (2015). Failure analysis of high-strength fiber ropes. Materials Science Forum, 825-826, 891-898.

Schmieder, A., Heinze, T., \& Michael, M. (2016). Computerassisted tomography analysis of high-strength fiber ropes. In Proceedings of the 6th conference on industrial computed tomography. Wels, Austria.

Smith, R., Nickerson, J., Palazzola, M. A., \& Pawrrish, R. (2005). Apparatus for testing aramid fiber elevator cables. Patent US 6923065 B2, USA.

Thévanaz, L., Niklès, M., Fellay, A., Facchini, M., \& Robert, P. (1998). Applications of distributed brillouin fibre sensing. In Proceedings of the international conference on applied optical metrology. Balatonfüred, Hungary.

Toda, M., Grabowska, K. E., \& Ciesielska-Wrobel, I. L. (2016). Micro-CT supporting structural analysis and modelling of ropes made of natural fibers. Textile Research Journal, 86(12), 1280-1293.

Törnqvist, R., Strande, M., Cannell, D., Gledhill, P., Smeets, P., \& Gilmore, J. (2011). SS: Deployment of subsea equipment: Qualification of large diameter fibre rope for deepwater construction applications. In Proceedings of the offshore technology conference. Houston, USA.

Uttamchandani, D., Culshaw, B., Overington, M. S., Parsey, M., Facchini, M., \& Thévanaz, L. (1999). Distributed optical fibre sensing in synthetic fibre ropes and cables. In Proceedings of the 13th international conference on optical fiber sensors. Kyongju, Korea.

Van Der Woude, F., \& Zijlmans, J. (2015). Real-time rope monitoring. Patent WO 2015160254 A1.

Vanderveldt, H. H., \& Tran, O. (1971). Acoustic emission from synthetic rope. Naval Engineers Journal, 83(6), 65-68.

Vlasblom, M. P., \& Bosman, R. L. M. (2006). Predicting the creep lifetime of HMPE mooring rope applications. In Proceedings of OCEANS. Boston, Massachusetts, USA. 
Wang, X., \& Chung, D. D. L. (1997). Real-time monitoring of fatigue damage and dynamic strain in carbon fiber polymer-matrix composite by electrical resistance measurement. Smart Materials and Structures, 6(4), 504-508.

Weller, S. D., Davies, P., Johanning, L., \& Banfield, S. (2013). Guidance on the use of synthetic fibre ropes for marine energy devices. Report from the MERiFIC Project, RDT CSM 13-232.

Weller, S. D., Davies, P., Vickers, A. W., \& Johanning, L. (2015). Synthetic rope responses in the context of load history: The influence of aging. Ocean Engineering, 96, 192-204.

Weller, S. D., Johanning, L., Davies, P., \& Banfield, S. J. (2015). Synthetic mooring ropes for marine renewable energy applications. Renewable Energy, 83, 12681278 .
Williams, J. H., Hainsworth, J. L., \& Lee, S. (1984). Acoustic-ultrasonic nondestructive evaluation of double-braided nylon ropes using the stress wave factor. Fibre Science And Technology, 21, 169-180.

Williams, J. H., \& Lee, S. (1982). Acoustic emission/rupture load characterizations of double-braided nylon rope. Marine Technology, 19(3), 268-271.

Winter, J. M., \& Green, R. E. (1985). Nondestructive evaluation of synthetic nylon rope using mechanical spectroscopy. In Proceedings of the 11th world conference on nondestructive testing. Las Vegas, Nevada, USA.

Yeager, L. L., Hochrein, A. A., \& Sherrard, J. R. (1982). Application of internal friction damping as a nondestructive evaluation technique for synthetic ropes used as spm hawsers in deepwater ports. In Proceedings of the offshore technology conference. Houston, Texas, USA. 